\title{
A Rare Case of Late LAD Reimplantation after Arterial Switch Operation
}

\author{
Yannick Kabulo Kolela $^{1,2}$, Maureen Klepper ${ }^{1,2}$, Geoffroy de Beco ${ }^{1,2}$, Thierry Sluysmans ${ }^{3}$ and \\ Alain Poncelet ${ }^{1,2, *}$ \\ ${ }^{1}$ Department of Cardiovascular and Thoracic Surgery, Cliniques Universitaires Saint-Luc, Brussels, Belgium \\ ${ }^{2}$ Pôle de Recherche Cardiovasculaire, Institut de Recherche Expérimentale et Clinique (IREC), Université Catholique de Louvain and \\ Division of Cardiothoracic and Vascular Surgery, Cliniques Universitaires Saint-Luc, Brussels, Belgium \\ ${ }^{3}$ Department of Pediatric Cardiology, University Hospital Saint-Luc, Brussels, Belgium \\ *Corresponding Author: Alain Poncelet. Email: alain.poncelet@uclouvain.be
}

Received: 26 May 2021 Accepted: 15 July 2021

\begin{abstract}
Arterial switch operation (ASO) is a complex neonatal operation in which transfer of the coronary arteries origins is the key to success. Coronary events after a successful ASO are not uncommon. We describe a rare case of a child who underwent an ASO in the neonatal period with one coronary (LAD) described as atretic left in place. At age seven, he developed myocardial ischemia due to retrograde flow with a steal phenomenon from the LAD into the pulmonary artery. The patient underwent a late LAD reimplantation. This case underscores that even very small ostia should be translocated at the time of ASO.
\end{abstract}

\section{KEYWORDS}

Late LAD reimplantation; arterial switch operation; transposition of the great arteries; congenital; surgery

\section{Introduction}

Transposition of the great arteries (TGA) is a congenital cardiac malformation characterized by atrioventricular concordance and ventriculo-arterial discordance [1]. In the past decades, the arterial switch operation (ASO) has become the preferred surgical procedure for transposition of the TGA in neonates. This operation is performed with a low mortality rate in many institutions. Successful transfer of the coronary arteries to the neoaorta represents the key-point of the procedure. The difficulty of this surgical step is greatly influenced by the variable coronary anatomy noted in patients with TGA. Coronary complications remain the main cause of mortality and morbidity following ASO [2]. We describe in this article, a rare case of late left anterior descending artery (LAD) reimplantation performed seven years after an ASO. This case presents a similar patho-physiology to the anomalous origin of left coronary artery from the pulmonary artery (ALCAPA) syndrome, characterized by a «coronary steal» phenomenon with a retrograde perfusion of LAD toward the pulmonary artery (due to low blood pressure in the pulmonary artery) resulting in left ventricular hypoperfusion [3]. 


\section{Case Presentation}

A 7-year-old boy was diagnosed with a transposition of the great arteries during the prenatal period. He benefited from the Rashkind maneuver at birth and underwent an ASO at 7 days of life in a foreign institution. Intraoperative aortic root exploration showed an atretic LAD contrasting with a dominant right coronary artery giving the circumflex with a posterior loop (1LAD-2RCx). Giving this anatomy, only the right coronary artery was reimplanted on the neoaorta. The postoperative course was uncomplicated, and the patient was then regularly followed. He became athletic. Beta-blocker's treatment was introduced to avoid the risk of sudden death. At the age of 7, a reverse flow in this "LAD" was seen. The patient was referred to our institution for a potential surgical correction.

The patient was asymptomatic. The physical examination at admission showed a systolic murmur $3 / 6$ maximum at the pulmonary focus and radiating into the back and no signs of heart failure was present. The electrocardiogram was normal, and the echocardiographic study showed a mild septal dyskinesia. The cardiac catheterization and the angio-CT demonstrated a rare coronary pattern: 1proximal LAD and 2-RCA-Cx-distal LAD pattern (posterior loop) and severe proximal stenosis of "LAD" arising from pulmonary artery (Figs. 1-4).

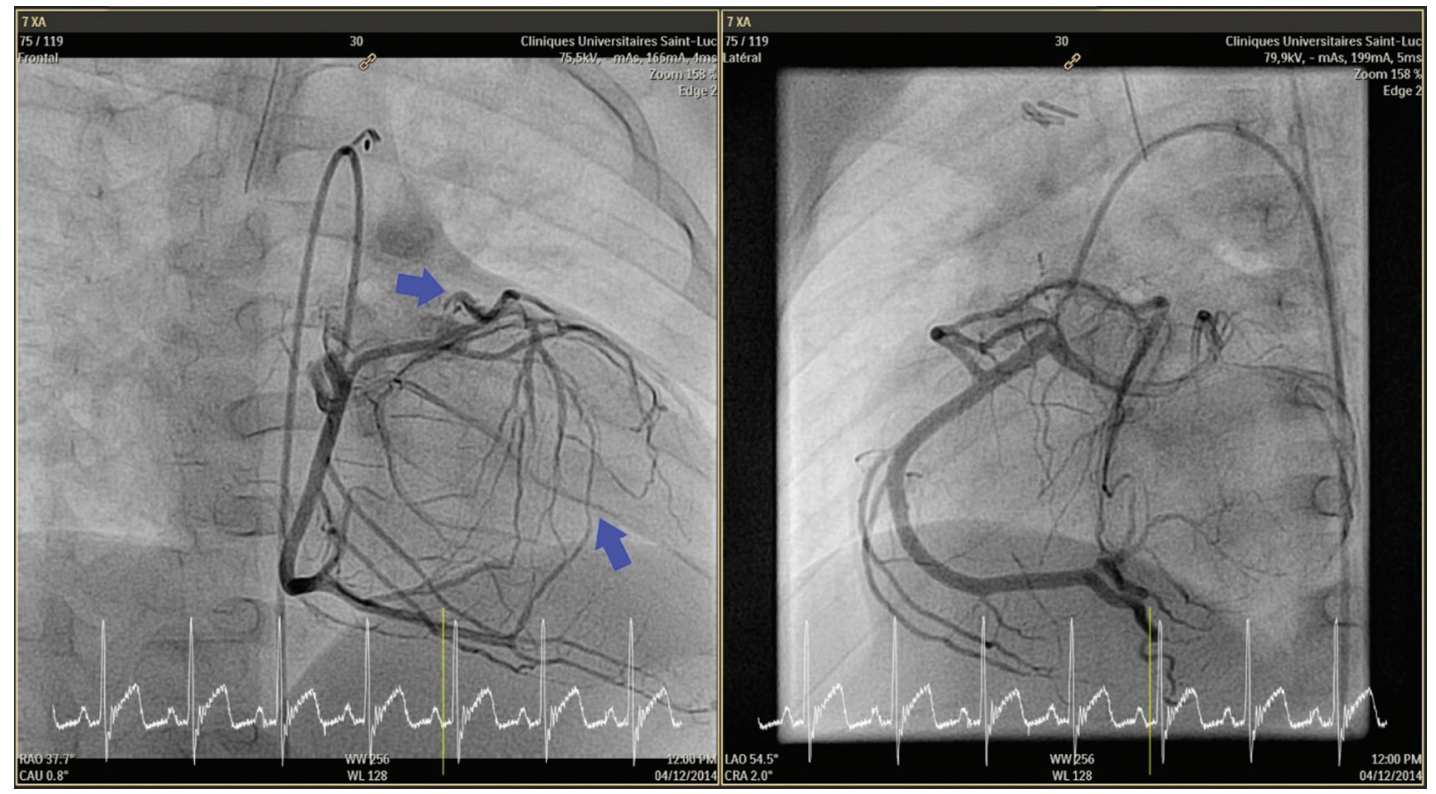

Figure 1: Cardiac catherization: RCA gives Cx. Reverse flow from multiples collaterals in the small LAD which originates from the pulmonary artery

A dobutamine stress echocardiogram showed an abnormal sub-endocardial perfusion at the septal level with a normal viability study. Given this clinical presentation and the risk of sudden death, LAD reimplantation was indicated.

After redo-sternotomy, normothermic cardiopulmonary bypass (CPB) was instituted by central canulation and bicaval venous drainage. Careful dissection of the ascending aorta and complete isolation of the overriding common pulmonary artery with its right and left branches was made with the beating heart. After vena cava exclusion, a transverse incision was made on the main pulmonary artery and the retroperfusing "LAD" was identified (sinus 1) with his proximal stenotic segment and multiple collateral epicardial vessels (Fig. 5). Excision of this accessory LAD with a small pulmonary wall flange was made and an additional mobilization of the vessel over $2 \mathrm{~cm}$ was performed. Excision of the proximal stenotic 
segment was made with a small posterior split and calibration of the vessel till $2 \mathrm{~mm}$ diameter. Direct closure of the harvest site on the CPA was made. The appropriate coronary reimplantation site on the left anterolateral side of the aorta was identified after adequate aortic root and heart filling. Excision of a $3 \mathrm{~mm}$ aortic wall flange was performed after aortic clamping and bulbar cardioplegia. The "LAD" was reimplanted with 8/0 polypropylene continuous suture (Fig. 6).

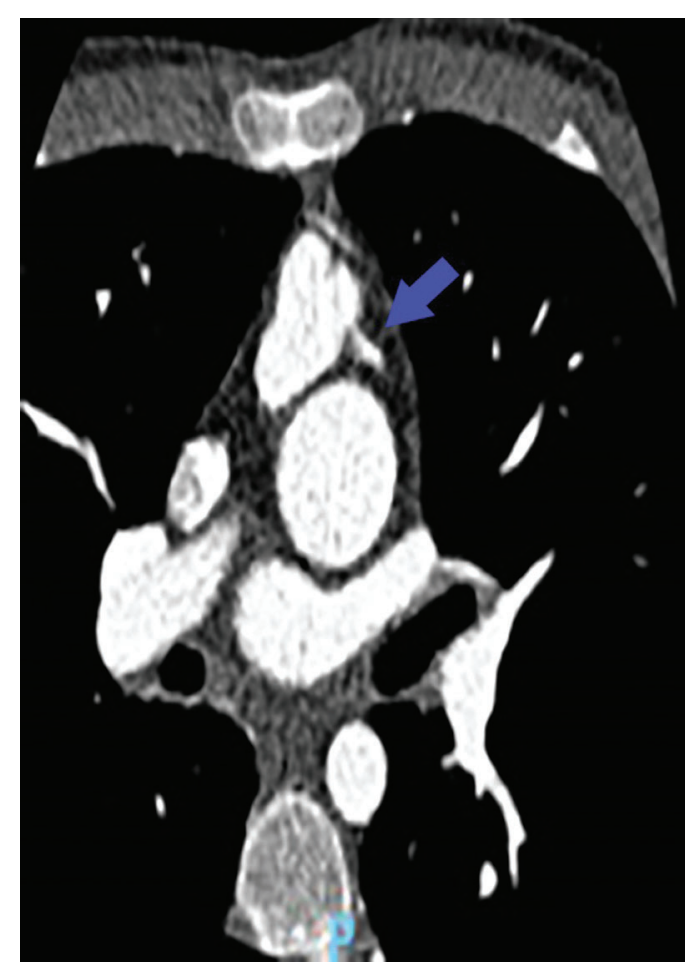

Figure 2: Angio-CT: LAD coronary arising from pulmonary artery

CPB weaning was easy with normal EKG, TEE showed good LV function with antegrade flow in the proximal LAD (Figs. 7 and 8). Postoperative course was uneventful, the patient was discharge on the $6^{\text {th }}$ postoperative day.

\section{Discussion}

This is a rare case of a child who had an ASO in the neonatal period with one coronary artery implantation and one coronary artery (LAD) described as atretic left in place. During infancy retrograde flow developed with a steal from the LAD into the pulmonary artery. This case has similar clinical presentation and patho-physiology to ALCAPA syndrome. In ALCAPA syndrome, myocardial ischemia results in the typical presentation of failure to thrive, profuse sweating, dyspnea, pallor in infancy, and later in childhood as atypical chest pain on eating or crying. In adults, malignant arrhythmias leading to sudden death may be the first manifestation of the anomaly [4]. In our case, the extensive collateral circulation between coronary vessels could explain the absence of symptoms at rest. However, stressinduced ischemia demonstrated at dobutamine echocardiogram motivated our therapeutic approach because of the lifelong risk of ischemia, ventricular arrythmias and sudden death if left untreated. This case shows the importance of successful coronary transfer in ASO. Elmahrouk et al. [5] reported a case of only one coronary artery transferred in ASO. In his case, the right coronary ostium was the only coronary transferred because the left coronary sinus had a blind indentation from which a firm cord-like 
left main coronary artery was originating. After one year of follow up no complications related to single coronary artery was reported. In our opinion, we should strive to reimplant both coronaries at the time of the ASO to reduce the risk of early and late coronary complications. The coronary artery anatomy in patients with TGA is a contributing factor for outcome in arterial switch procedure. However, abnormal coronary arterial pattern does not impede ASO to be done successfully. Many techniques of coronary artery transfer have been described [6,7]. Whenever the arterial switch is not feasible, alternative approaches are required [1]. Even after a successful arterial switch operation, coronary events including stenosis, significant obstruction or complete obliteration are known as early and late complications. Late coronary stenosis or occlusion is not infrequent $(0,3-11,3 \%)[2,8,9]$. These patients remain generally asymptomatic and the first manifestation of the coronary abnormality maybe circulatory collapse or unexpected sudden death [8].

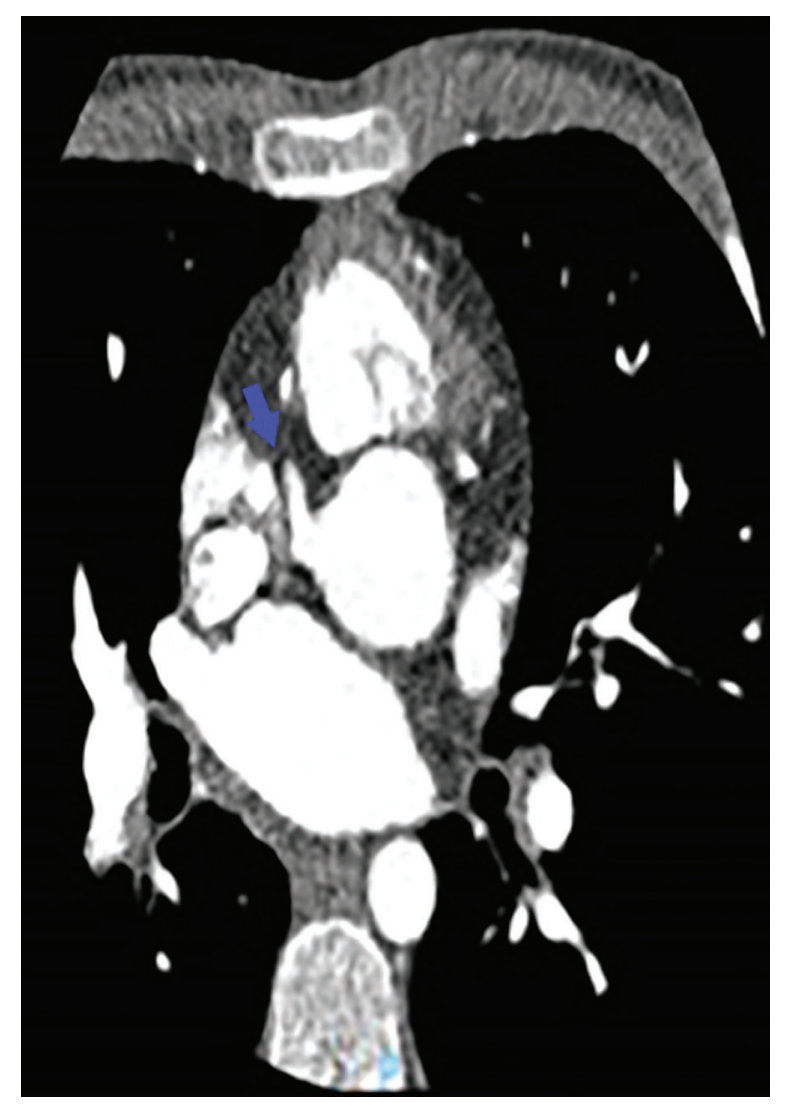

Figure 3: Angio-CT: Only the right coronary artery arises from the aorta 


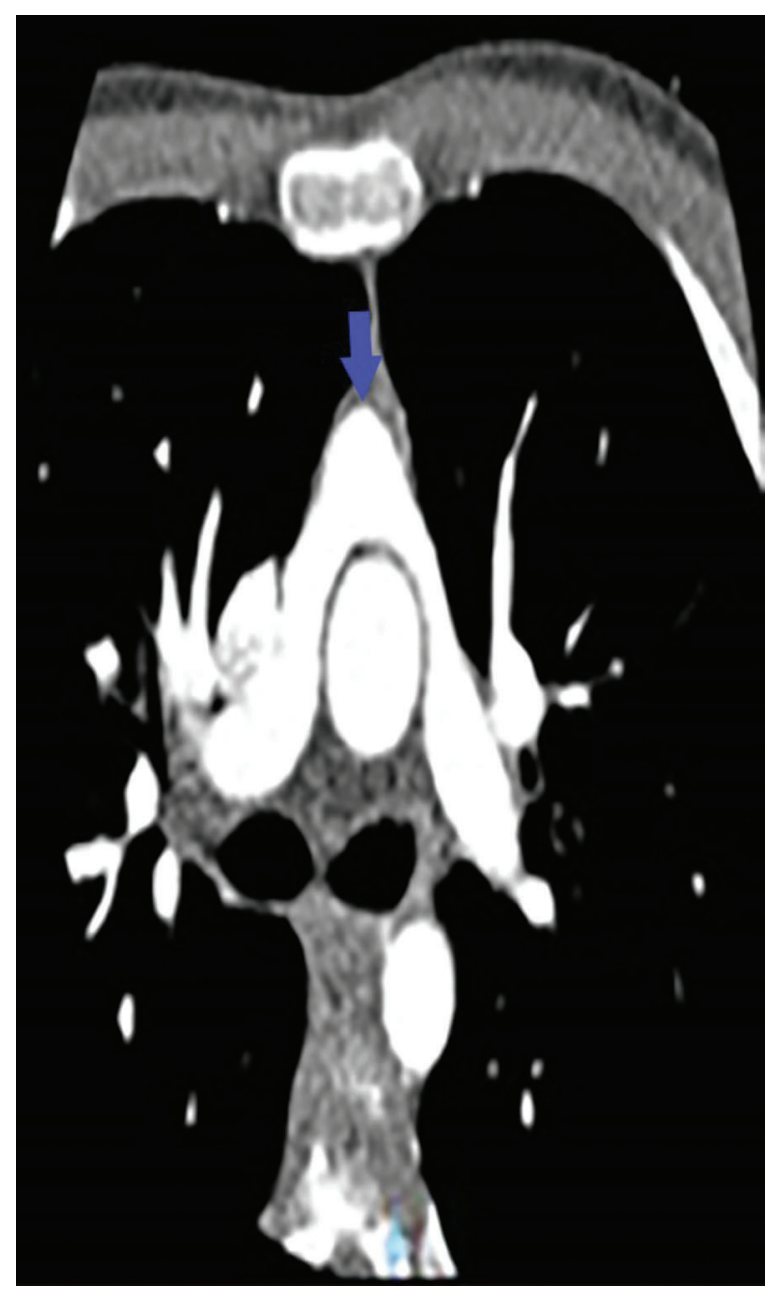

Figure 4: Angio-CT: Pulmonary artery with its two branches in front of the aorta testifying the Lecompte maneuver performed during the arterial switch operation

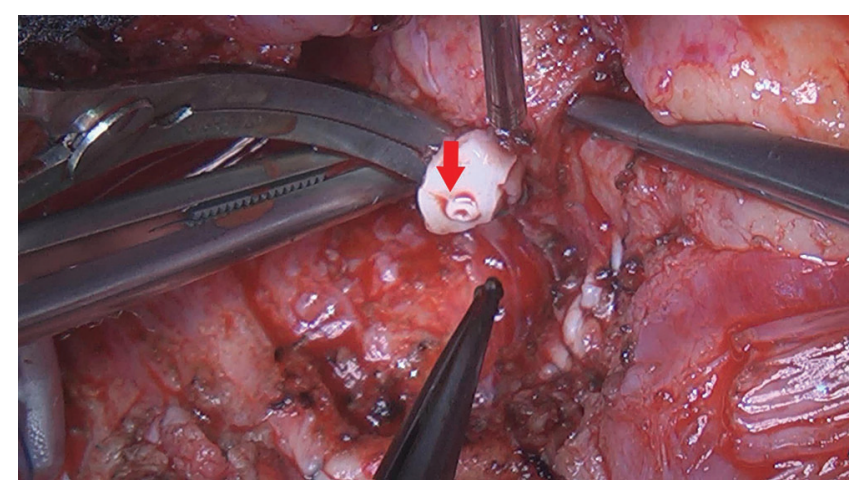

Figure 5: Intraoperative views: Stenotic proximal segment of the accessory LAD 


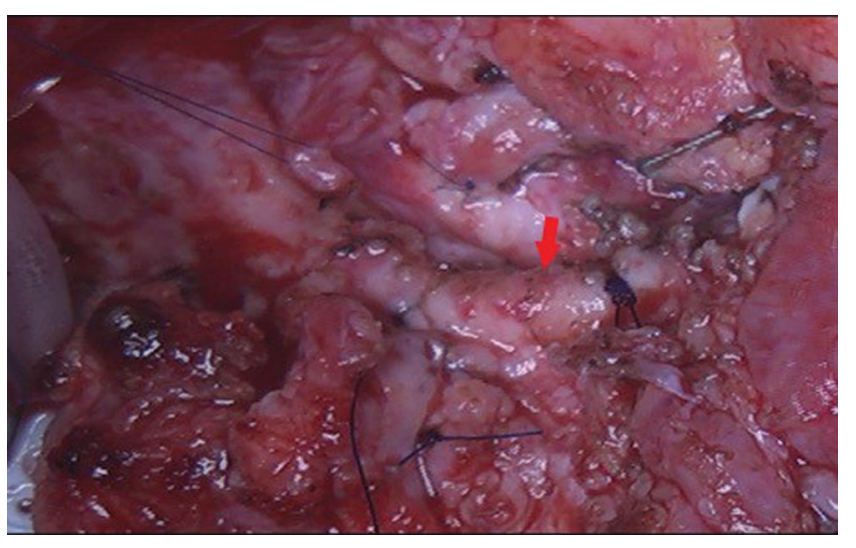

Figure 6: Surgical view after LAD reimplantation in the aorta

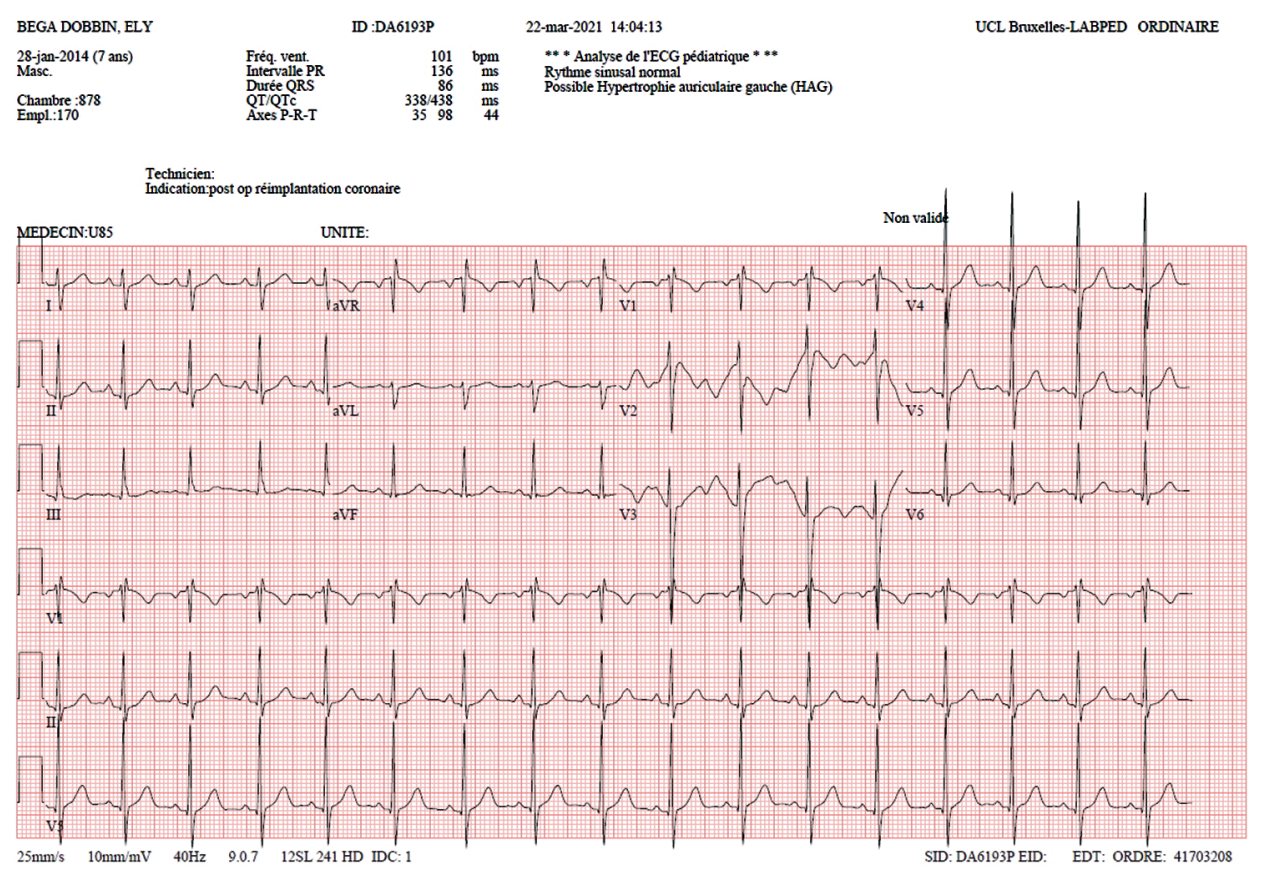

Figure 7: EKG: No signs of ischemia 


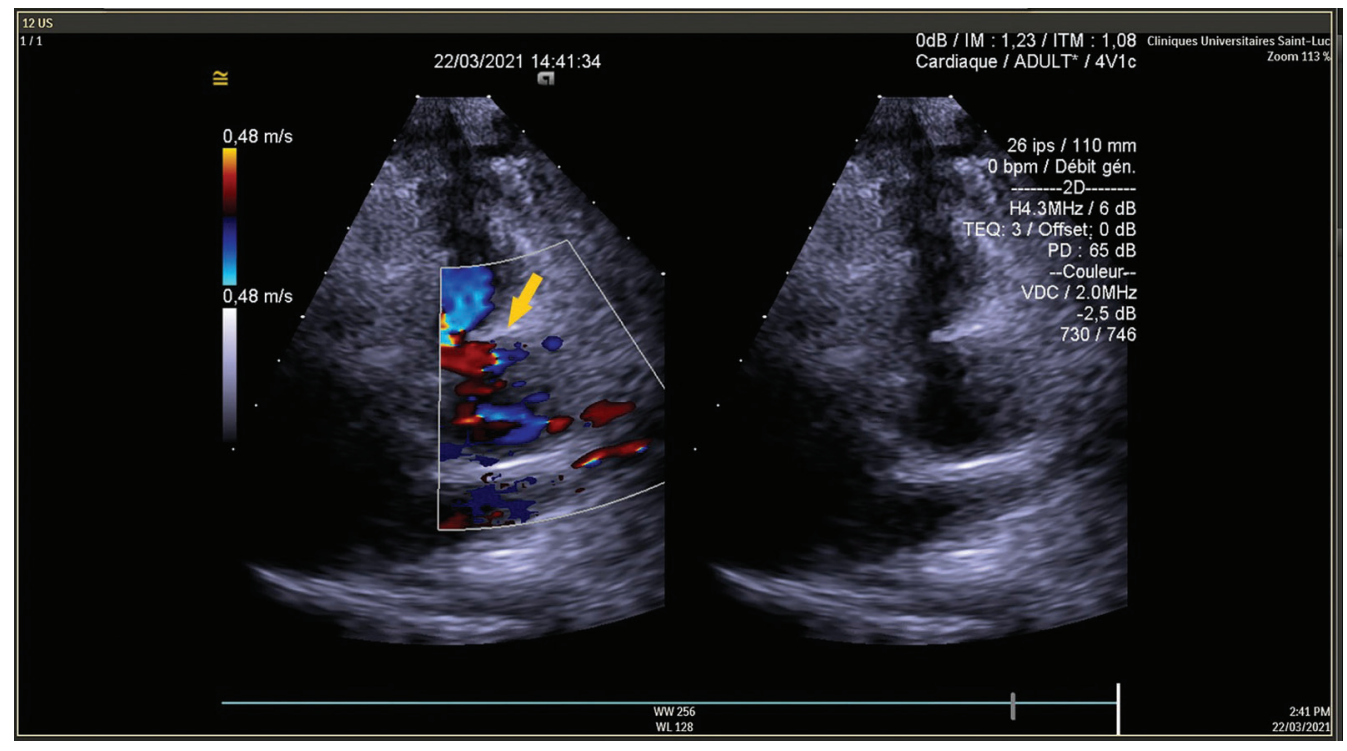

Figure 8: TEE shows antegrade flow in LAD

\section{Conclusion}

To our knowledge, this is the first case of a late LAD reimplantation following an ASO. Myocardial ischemia due to coronary artery lesions (stenosis or complete obliteration) after ASO is common but in this case, myocardial ischemia was a consequence of an hypoplastic LAD left in a place at the time of ASO. This case report illustrates that even very small ostia should be translocated at the time of arterial switch operation.

Ethics Statements: Patient consent for publication is obtained.

Funding Statement: The authors received no specific funding for this study.

Conflicts of Interest: The authors declare that they have no conflicts of interest to report regarding the present study.

\section{References}

1. Martins, P., Castela, E. (2008). Transposition of the great arteries. Orphanet Journal of Rare Diseases, 3, 27.

2. Tamisier, D., Ouaknine, R., Pouard, P., Mauriat, P., Lefebvre, D. et al. (1997). Neonatal arterial switch operation: Coronary artery patterns and coronary events. European Journal of Cardio-Thoracic Surgery: Official Journal of the European Association for Cardio-thoracic Surgery, 11(5), 810-817.

3. Peña, E., Nguyen, E. T., Merchant, N., Dennie, C. (2009). ALCAPA syndrome: Not just a pediatric disease. RadioGraphics, 29(2), 553-565. DOI 10.1148/rg.292085059.

4. Frapier, J. M., Leclercq, F., Bodino, M., Chaptal, P. A. (1999). Malignant ventricular arrhythmias revealing anomalous origin of the left coronary artery from the pulmonary artery in trio adults. European Journal of Cardio-Thoracic Surgery, 15(4), 539-541.

5. Elmahrouk, A. F., Hamouda, T., Ismail, M. F., Jamjoom, A. (2018). Transposition of great arteries with left main coronary artery atresia-case report. Thoracic and Cardiovascular Surgeon Reports, 7(1), e16-e17.

6. Yacoub, M. H., Radley-Smith, R. (1978). Anatomy of the coronary arteries in transposition of the great arteries and methods for their transfer in anatomical correction. Thorax, 33(4), 418-424.

7. Aubert, J., Pannetier, A., Couvelly, J. P., Unal, D., Rouault, F. et al. (1978). Transposition of the great arteries. New technique for anatomical correction. British Heart Journal, 40(2), 204-208. 
8. Tsuda, T., Bhat, A. M., Robinson, B. W., Baffa, J. M., Radtke, W. (2015). Coronary artery problems late after arterial switch operation for transposition of the great arteries. Circulation Journal: Official Journal of the Japanese Circulation Society, 79(11), 2372-2379.

9. Santens, B., van de Bruaene, A., de Meester, P., Gewillig, M., Troost, E. et al. (2020). Outcome of arterial switch operation for transposition of the great arteries. A 35-year follow-up study. International Journal of Cardiology, 316, 94-100. 